\title{
An energy-efficient adaptive frameless ALOHA protocol
}

\author{
Samira Rahimian, Moslem Noori* (D) and Masoud Ardakani
}

\begin{abstract}
Random access protocols are a key feature of a family of emerging communication networks such as machine-to-machine, radio frequency identification (RFID), and sensor networks. To accommodate the needs of such networks with a massive number of uncoordinated devices, new random multiple access (MAC) protocols have been proposed that aim to improve the system efficiency by resolving collisions in the received signal. In this work, we consider one of such protocols, called frameless ALOHA, and propose two techniques to improve its energy efficiency without sacrificing the network throughput. More specifically, we propose mechanisms to adaptively control the access probability at the users. The proposed mechanisms are local and like the original frameless ALOHA, no coordination between the users is needed. Our simulation results verify the improvement achieved in the energy efficiency by the proposed techniques.
\end{abstract}

Keywords: Random access protocol, ALOHA, Energy efficiency, Throughput, Interference cancellation

\section{Introduction}

Most communication networks consist of a number of users sharing a common medium (channel) for data communication. Depending on the requirements of the users and available resources of the network, two types of medium access control (MAC) protocols have been mainly employed to facilitate the users' access to the common channel. In the first type of MAC protocols, users share the medium in an organized predetermined fashion such that interference is fully avoided at the network. However, in the second approach, users share the medium in a random and distributed manner which makes it more flexible compared to the first approach at the price of occasional collisions in the network. Moreover, random MAC protocols make better use of the network resources when users have sporadic traffic behavior.

Among the random MAC protocols for wireless systems, ALOHA [1] and its modified versions have been widely used for many wireless setups. Accommodating the needs of emerging wireless applications (e.g., radio frequency identification (RFID), machine-tomachine (M2M), and wireless sensor networks), however, poses new challenges in MAC design and further modifications on ALOHA are needed [2, 3]. More specifically, in such networks, there often exist a large number of uncoordinated battery-powered devices with power resources. This necessitates devising random MAC protocols that are energy-efficient yet have high enough throughput to handle the traffic load. To this end, many studies have investigated the ways to improve the throughput and/or energy efficiency of the original ALOHA protocol to meet the demands of these networks. In this paper, we focus on further advancing the existing results on high-throughput energy-efficient ALOHA random access protocols.

The organization of the rest of the paper is as follows. An overview on the state of the art of ALOHA protocols is presented in Section 2. The contributions of this paper are summarized in Section 3. Then, Section 4 formally introduces the system model for frameless slotted ALOHA as the basis of our work. Our proposed adaptive transmission strategies to improve the energy efficiency of frameless slotted ALOHA is discussed in Section 5. Simulation results are presented in Section 6. Finally, Section 7 concludes the paper.

*Correspondence: moslem@ualberta.ca

Department of Electrical and Computer Engineering, University of Alberta,

Edmonton, Canada

\section{Springer Open}

(c) 2016 The Author(s). Open Access This article is distributed under the terms of the Creative Commons Attribution 4.0 International License (http://creativecommons.org/licenses/by/4.0/), which permits unrestricted use, distribution, and reproduction in any medium, provided you give appropriate credit to the original author(s) and the source, provide a link to the Creative Commons license, and indicate if changes were made. 


\section{State of the art on ALOHA protocol}

The first version of ALOHA, also known as pure ALOHA, is a distributed random access in which each user transmits whenever it has a packet for transmission. There is no time slot in pure ALOHA, and all the packets are of the same size. Successful transmissions are the ones that do not bare any interference. The achievable throughput, $T$, defined as the average number of successful packet transmissions per packet time, in this scheme is $\frac{1}{2 e}$.

The introduction of slotted [4] and framed slotted [5] ALOHA was among the first attempts to improve the throughput of pure ALOHA protocol. In slotted ALOHA, the shared access time is divided into slots of equal duration. Users are only allowed to send their packets at the beginning of a time slot. Only collision free packets, i.e., the ones that are not interfered by packets transmitted by other users, are counted as successful transmissions. This random access scheme doubles the achievable throughput of pure ALOHA reaching a throughput of $\frac{1}{e}$.

Framed slotted ALOHA [5] is a version of slotted ALOHA where the shared access time is divided into frames, consisting of $M$ time slots. Each user may have a transmission in a frame with probability $p$, called the access probability. Every user who intends to transmit randomly and uniformly selects one of the time slots in the frame to transmit its packet. Same as the slotted ALOHA scheme, only collision-free slots are considered as successful transmissions in a frame. The maximum throughput of the framed slotted ALOHA is also $\frac{1}{e}$. However, framed slotted ALOHA has a lower implementation complexity compared to slotted ALOHA since the synchronization among the users is on the basis of a frame rather than a time slot.

In an attempt to further improve the throughput of the framed slotted ALOHA, the authors in [6] propose the idea of transmission of several replicas of the same user message within a frame. This idea was further developed in [7], by introducing successive interference cancellation (SIC) technique which considerably increases the throughput of the framed slotted ALOHA. To this end, the receiver first resolves the collision-free packets within a frame. Then, the corresponding interferences of the these packets in other slots are canceled. By doing such, some other time slots may become interference-free which in turn results in resolving new packets. The process of resolving packets through successively canceling the interference continues until either no more time slot becomes interference-free or all the packets in that frame are resolved.

The idea of repeating users' messages was later improved through irregular repetition method [8] where the number of replicas sent by the users follows a specific probability distribution function. By optimizing this probability distribution function, it is shown in [8] that the throughput of the framed slotted ALOHA can be even further improved.

More recently in [9], a new technique, called frameless ALOHA, has been developed. Unlike conventional framed slotted ALOHA protocols and inspired by the concept of rateless coding [10], frameless ALOHA avoids using frames with a fixed number of slots. Instead, users attempt to transmit their packets during consecutiveve contention rounds that can be seen as frames with variable number of slots. The length of each contention round is enforced by stopping criteria that are defined based on the number of resolved packets and the instantaneous throughput at the destination. Using such a dynamic approach for adjusting the length of contention rounds and employing SIC at the destination, frameless ALOHA increases the network throughput, and consequently its energy efficiency, compared to the previous variations of ALOHA protocol. Frameless slotted ALOHA is the base of our work in this paper and is explained further in detail later.

Similar to ALOHA throughput improvement, different approaches have been proposed to improve the energy efficiency of (framed) slotted ALOHA [11-14]. For instance, optimizing the frame size [11] and nodes' access (transmission) probabilities [12] are ways to improve the energy efficiency of slotted ALOHA. The work reported in [13] also suggests a probabilistic approach, called tree slotted ALOHA, to lower the number of transmissions in the system which consequently results in energy saving. In this work, we attempt to improve the energy efficiency of ALOHA protocol via exploiting adaptive access probabilities at the users. Details of our contribution are outlined in the following section.

\section{Summary of contributions}

The focus of this paper is on improving the energy efficiency of frameless slotted ALOHA which is one of the best performing ALOHA protocols in terms of throughput. Frameless ALOHA is also highly decentralized; thus, improving its energy efficiency would make it even more suitable for applications in M2M, RFID, and sensor networks.

To be more specific, we propose two adaptive frameless slotted ALOHA protocols to maximize the energy efficiency of the access strategy while a desired throughput is guaranteed. The basic idea behind our proposed schemes is to adaptively change the access probability of the users. That is, all users start with the same access probability at the start of the contention round. Later, each user locally updates its current access probability based on whether it has transmitted in the previous time slot or not. For this, if the user transmitted in the previous time slot, it decreases its current access probability and increases the access probability otherwise. The rationale behind this strategy is to give a higher chance to the users that have 
not transmitted previously and protect them from the possible interference caused by the users that have already made a transmission.

The difference between our two proposed schemes is in the step size by which the access probability changes adaptively. While the first proposed scheme employs a fixed step size, the second one benefits from variable step sizes. To further improve the performance of the suggested schemes, optimizing the step sizes are also considered. In fact, to achieve the best energy efficiency performance, we jointly optimize the stopping criteria and initial access probability of the frameless ALOHA and the step sizes of our proposed schemes.

As we discuss later in the paper, in the second proposed approach, by setting a simple relation between the increase and decrease step sizes, but letting them to change from one time slot to another, we are able to simplify the optimization problem yet surprisingly further improve the energy saving. Numerical results show that our proposed schemes significantly improve the energy efficiency of the network by reducing the average number of transmissions per time slot. In some cases, the reported energy saving reaches an outstanding value of $20 \%$ when a variable step size is used. It is worth mentioning that our proposed approaches are distributed and such performance improvement is achieved without extra cost of central monitoring or hand shaking between the users and the base station (BS).

\section{System model}

We assume that there are $N$ users in the network, denoted by $u_{i}$ where $i \in \mathcal{N}=\{1,2, \ldots, N\}^{1}$. Users always have packets ready for transmission to the BS and employ frameless slotted ALOHA as the access protocol to share the channel. Like any other slotted ALOHA protocol, in frameless slotted ALOHA, the access time to the channel is divided into equal duration slots. The length of each slot is equal to the time needed to transmit a packet plus a possible guard time to counteract propagation delays [15]. Similar to the original slotted ALOHA protocol, users are synchronized on the slot basis for example via using a global synchronization mechanism [4].

However, opposed to framed ALOHA, users' transmissions do not happen within fixed size frames in frameless ALOHA. Instead, users attempt to transmit their packets during one contention round that is a set of time slots where users are permitted to have their transmissions. Here, we denote the number of time slots in a contention round by $M$, which is not a priori determined and can vary from one contention round to another. In each slot of the contention round, say slot $m$, each user, say $u_{i}$, attempts to transmit its packet by probability $p_{i}(m)$. Users continue their transmissions until they receive a beacon message from the BS notifying them about the end of the contention round ${ }^{2}$. Through the feedback from the BS at the end of contention round, users are also informed whether their packets have been successfully received by the BS or not. Users whose packets have not been resolved in a contention round may attempt to transmit their packets in the following contention round. Later in this section, we will discuss the stopping criteria used by the BS to terminate a contention round and start a new one.

Following the users' transmissions in a contention round, each arbitrary time slot has one of the following statuses: (a) no transmission (idle slot), (b) only one transmission (singleton slot), (c) more than one transmission (collision slot). To improve the throughout performance of the system, BS exploits SIC to resolve some of the packets received during the collision slots. To this end, the BS needs to store the received signals in the previous slots of a contention round. After the arrival of new time slot, the BS executes a round of SIC where it first resolves the collision-free packets from the stored and current received signals. Then, the $\mathrm{BS}$ cancels the corresponding interference of the resolved packets from collision slots. By doing such, some other singleton slots may appear making resolving some other packets possible. This process is iteratively repeated until no more packets can be resolved. At this point, the BS either terminates the contention round if the stopping criteria is met or waits for the next time slot to resolve further packets.

A toy example of SIC execution is shown in Fig. 1. In this graphical representation, there are four time slots in the contention round. Users are on the left side of the graph and time slots are on the right side of the graph. In this graph, a user is connected to a slot if it has transmitted a packet during that time slot. First singleton happens in time slot $s_{2}$ which leads to resolving $u_{1}$ packet. Now, by canceling the interference of $u_{1}$ 's packet from $s_{4}, s_{4}$ becomes a singleton slot from which $u_{2}$ 's packet can be resolved. Eventually, by canceling the interference of $u_{2}$ 's packet from $s_{1}, u_{3}$ 's packet is resolved.

To explain the stopping criteria of the contention rounds, we first formally model the users' transmissions. Assume that $u_{i}$ wants to send its message, $X_{i}$, to the BS in a contention round. The received signal in time slot $m$ of the contention round at the BS, denoted by $Y_{m}$, can be modeled as ${ }^{3}$

$$
Y(m)=\sum_{i=1}^{N} s_{i}(m) X_{i} \quad \forall m \in \mathcal{M},
$$

where $s_{i}(m)$ is a Bernoulli random variable indicating whether $u_{i}$ has transmitted in time slot $m$ or not, i.e., $s_{i}(m)=1$ if transmission has occurred and $s_{i}(m)=0$ if it has not. Also, $\mathcal{M} \triangleq\{1,2, \ldots, M\}$. From the protocol description in the above, we have

$$
\mathrm{P}\left[s_{i}(m)=1\right]=p_{i}(m) \quad \forall i \in \mathcal{N}, m \in \mathcal{M},
$$




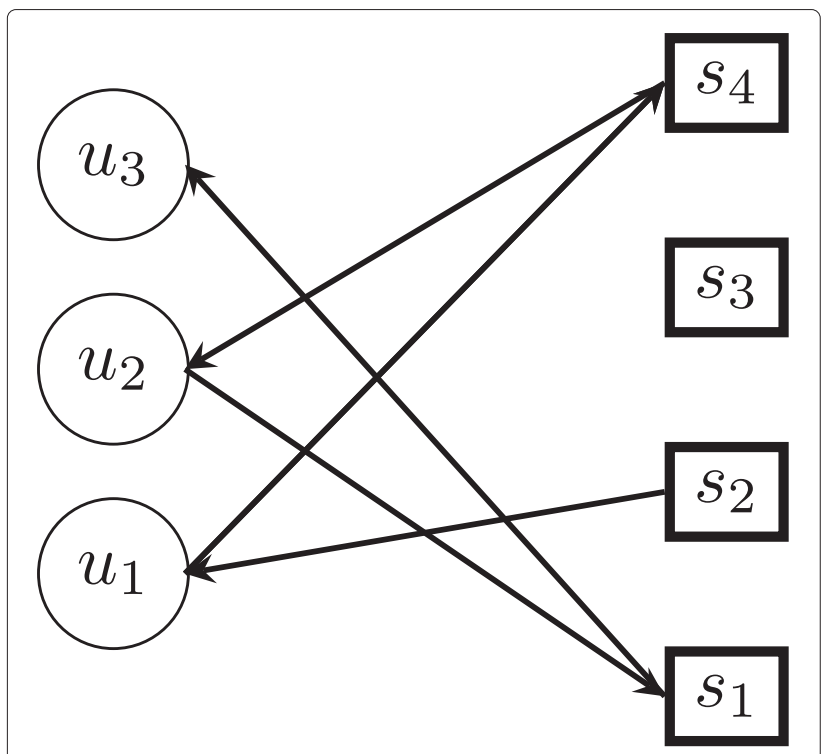

Fig. 1 Graph representation of SIC procedure

where $\mathrm{P}[\cdot]$ refers to the probability of an event.

After receiving each $Y(m)$, the BS executes SIC on $Y(m)$ plus all stored signals from previous time slots to resolve new packets. We define the instantaneous throughput at time slot $m$, called $T_{\mathrm{I}}(m)$, as the ratio of the number of resolved users' messages until a given time slot $m$, denoted by $N_{\mathrm{R}}(m)$, to the number of elapsed time slots. In other words,

$$
T_{\mathrm{I}}(m)=\frac{N_{\mathrm{R}}(m)}{m} .
$$

Clearly, at the end of a contention round, the achievable throughput, $T$, is equal to $T_{\mathrm{I}}(M)$. Similarly, the fraction of resolved users at time slot $m, F_{\mathrm{R}}(m)$, is defined as

$$
F_{\mathrm{R}}(m)=\frac{N_{\mathrm{R}}(m)}{N} .
$$

Now, the fraction of the resolved users at the end of a contention round is $F_{\mathrm{R}}=F_{\mathrm{R}}(M)$.

Now, we explain the stopping criteria based on $T_{\mathrm{I}}(m)$ and $F_{\mathrm{R}}(m)$ for ending a contention round at the BS. Andor-tree evaluations [16] of the frameless slotted ALOHA in [17] show that $T_{\mathrm{I}}(m)$ and $F_{\mathrm{R}}(m)$ both have an avalanche behavior versus the number of elapsed time slots. That is, a sudden jump from a low value to a much higher value is observed at a specific time slot. This comes from the inherent nature of SIC where suddenly a large number of users' transmissions are resolved by adding a single equation ${ }^{4}$. Further, the authors in [17] have shown that the avalanche point happens at the same time slot for both $T_{\mathrm{I}}(m)$ and $F_{\mathrm{R}}(m)$ most of the time. In addition, after the avalanche point, resolving the users' packets happens very slowly causing only marginal increment in $T_{\mathrm{I}}(m)$ and $F_{\mathrm{R}}(m)$. As a result, it is concluded that the throughput achieves its maximum right after the avalanche point. The only case that this does not hold is when $T_{\mathrm{I}}(m)=1$. In this case, $T_{\mathrm{I}}(m)$ reaches its maximum value, while $F_{\mathrm{R}}(m)$ may not be at its peak. That said, the stopping criteria to terminate a contention round is when $T_{\mathrm{I}}(m)=1$ or $F_{\mathrm{R}}(m)$ reaches a threshold $F$. To ensure the maximum achievable throughput, in the original frameless ALOHA, $F$ is found through a numerical optimization for different values of $N$ as discussed in [17]. The optimized value for $F$ is named $F_{\text {opt }}$.

\section{Modified frameless slotted ALOHA}

In this section, we propose two new ALOHA schemes. Both of these schemes aim at improving the energy efficiency of the original frameless ALOHA protocol [17] without compromising the throughput. In the following, we first give the general intuition behind our schemes and then provide the details of these schemes later in this section.

Note that in the original frameless ALOHA protocol $[9,17], p_{i}(m)=p_{\text {init }}$ for any $i$ and $m$, where $p_{\text {init }}$ is a fixed probability assigned to the users by the $\mathrm{BS}$. The value of $p_{\text {init }}$ is determined based on BS's estimation from the number of contending users $N$ in a contention round [17]. To improve the energy efficiency, our proposed schemes benefit from adaptive access probabilities at the users. Similar to the original frameless ALOHA, the BS sends a beacon message to the users indicating the start of a contention round, as well as their initial access probability $p_{\text {init. }}$ That is,

$$
p_{i}(1)=p_{\text {init }}, i \in \mathcal{N}
$$

After the first time slot, as time passes, each $u_{i}$ adaptively changes $p_{i}(m)$ in the following time slots of the contention round. More specifically, if the user had a transmission in the current time slot, it reduces its access probability for the next time slot. Also, if the user did not have a transmission in the current time slot, it increases its access probability for the next time slot. The intuition behind this approach is to increase the chance of successful transmission for those users that have not transmitted in the previous time slot by reducing the chance of collision from the users that already have transmitted. Note that for this to happen, the increase step should be smaller than the decrease step. This is to avoid many users having high access probabilities and thus to prevent having many collisions. While both proposed schemes adaptively change the access probability at the users to achieve energy efficiency, they follow different strategies. In the first approach, the steps are fixed over the whole contention round while in the second approach the step size is variable over different time slots. 


\subsection{Adaptive probability of access with fixed step size}

In this scheme, we assume that the increase and decrease steps at all the users and in all time slots are $\alpha$ and $\alpha k^{5}$, respectively, where $k$ is a positive integer. Similar to $p_{\text {init }}$, both $\alpha$ and $k$ are sent to the users by the BS at the start of a contention round. That said, the access probability of an arbitrary user $u_{i}$ at time slot $m \geq 2$ is updated as follows:

$$
p_{i}(m)= \begin{cases}\max \left\{p_{i}(m-1)-\alpha k, 0\right\} & \text { if } s_{i}(m-1)=1 \\ \min \left\{p_{i}(m-1)+\alpha, 1\right\} & \text { if } s_{i}(m-1)=0 .\end{cases}
$$

In (6), the max and min functions are to ensure that $p_{i}(m+1)$ stays within $[0,1]$. One should note that for any two users $u_{i}$ and $u_{j}$ and an arbitrary time slot $m, p_{i}(m)$ and $p_{j}(m)$ are identically distributed random variables. This is because these two users start from equal initial access probabilities and have equal step sizes for increasing or decreasing their access probabilities over time. Further, $\mathrm{E}\left[p_{i}(m)\right]=\mathrm{E}\left[p_{j}(m)\right]=\bar{p}(m)$ where $\mathrm{E}[\cdot]$ refers to the expected value and $\bar{p}(m)$ is the average access probability of the users at time slot $m$.

To better explain the scheme, we provide the flowchart representation of the scheme in Fig. 2. The stopping criteria in our adaptive version of frameless ALOHA is the same as the original frameless scheme, meaning that the BS stops a contention round either if the fraction of resolved users reaches a desired threshold, $F_{\mathrm{R}}$, or if the instantaneous throughput reaches 1 .

Now that we have explained our approach, we are interested in improving its performance by optimizing the design parameters of the network, i.e., $p_{\text {init }}, F, \alpha$, and $k$. This optimization is done at the BS whose goal is to minimize the energy consumption of the network while achieving a required throughput $T_{\text {req. }}$. To make sure that our scheme is an improvement to the original frameless ALOHA, we want $T_{\text {req }}$ not to be worse than the maximum throughput of the original frameless ALOHA, denoted by $T_{\mathrm{o}}$.

Instead of using the consumed energy as the objective function of our optimization problem, we can focus on minimizing the average number of transmissions by a user during a contention round. Note that the consumed energy within the network is proportional to the average number of transmissions and this parameter works as a relatively accurate measure for the energy consumption in the network. To measure the energy consumption through the number of transmissions, we first define user degree $d(i)$. This parameter represents the number of message replicas sent by an arbitrary user $u_{i}$ during a contention round. That is,

$$
d(i)=\sum_{m=1}^{M} s_{i}(m), \quad \forall i \in \mathcal{N} .
$$

Thus, the average number of transmissions per user in a contention round, namely $d_{N}$, is

$$
d_{N}=\frac{\sum_{i=1}^{N} d(i)}{N} .
$$

Now, we formulate the following optimization problem to minimize the energy consumption in the network while ensuring a throughout as good as the original frameless ALOHA

$$
\begin{array}{ll}
\underset{\alpha, k, p_{\text {init }}, F}{\min } & d_{N} \\
\text { s.t. } & T \geq T_{\mathbf{o}} .
\end{array}
$$

The optimal solution of this problem minimizes the average number of transmissions needed by each user to guarantee a throughput of $T_{\mathrm{o}}$. We denote the optimal solution of the above problem by $\alpha_{\text {opt }}, k_{\text {opt }}, p_{\text {initopt }}$, and $F_{\text {opt }}$. Further, the throughput achieved by the adaptive fixed step size approach and this set of parameters is called $T_{\mathrm{a}, \mathrm{f}}$. Due to its underlying complex form, we are unable to find an analytical solution for the above optimization problem. However, the problem can be solved numerically. Details of this numerical solution is presented in Section 6. Note that this optimization problem is solved at the BS and then $\alpha_{\mathrm{opt}}, k_{\mathrm{opt}}$, and $p_{\text {init }}$ opt are sent to the users at the start of a contention round.

\subsection{Analysis of the average access probability}

One way to achieve a better energy efficiency than the original frameless ALOHA is to ensure that on average, users transmit packets with lower probabilities than that of the original frameless ALOHA. This in turn translates into a lower number of transmissions in the network. For instance, let us assume that in both original and adaptive frameless ALOHA, users start from identical access probabilities at the start of a contention round. Now, if in the adaptive scheme, the average of the users' access probabilities is decreasing in $m$, i.e., $\bar{p}(m)<\bar{p}(m-1)$, we are guaranteed to have less number of transmissions compared to the original frameless ALOHA. That said, in the following, we present the analysis of $\bar{p}(m)$ and find a condition to enforce $\bar{p}(m)<\bar{p}(m-1)$. As we will see, satisfying this condition using fixed step sizes to adjust the access probabilities is very difficult. This would explain our motivation to propose second adaptive frameless ALOHA scheme featuring variable step sizes.

For the simplicity of presentation, let us first focus on $\bar{p}(1)$ and $\bar{p}(2)$ at an arbitrary user (see Fig. 3). Since the original access probability of all users is $p_{\text {init }}$, we have $\bar{p}(1)=p_{\text {init }}$. Now, if the contention round does not end after the first time slot (i.e., $T_{\mathrm{I}}(1) \neq 1$ ), the access probability in the second time slot has two possible values: it 


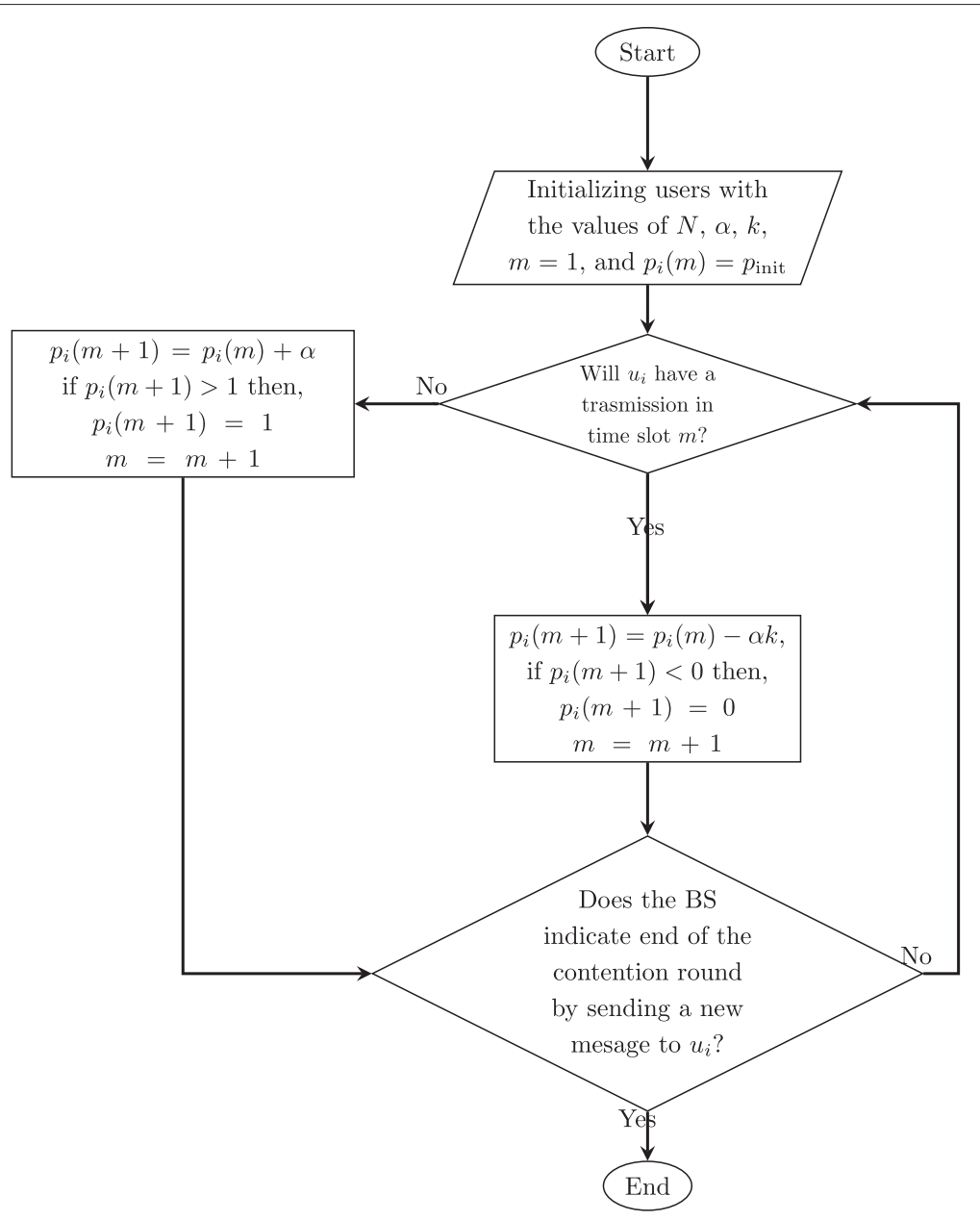

Fig. 2 Adaptive access strategy with fixed step size

is either $p_{\text {init }}-\alpha k$ if a transmission in the first time slot is made with probability $p_{\text {init }}$, or $p_{\text {init }}+\alpha$ with probability $1-p_{\text {init. }}$ Thus,

$$
\begin{aligned}
\bar{p}(2)= & \left(1-\mathrm{P}\left[T_{\mathrm{I}}(1)=1\right]\right)\left(p_{\text {init }}\left(p_{\text {init }}-\alpha k\right)\right. \\
& \left.+\left(1-p_{\text {init }}\right)\left(p_{\text {init }}+\alpha\right)\right) .
\end{aligned}
$$

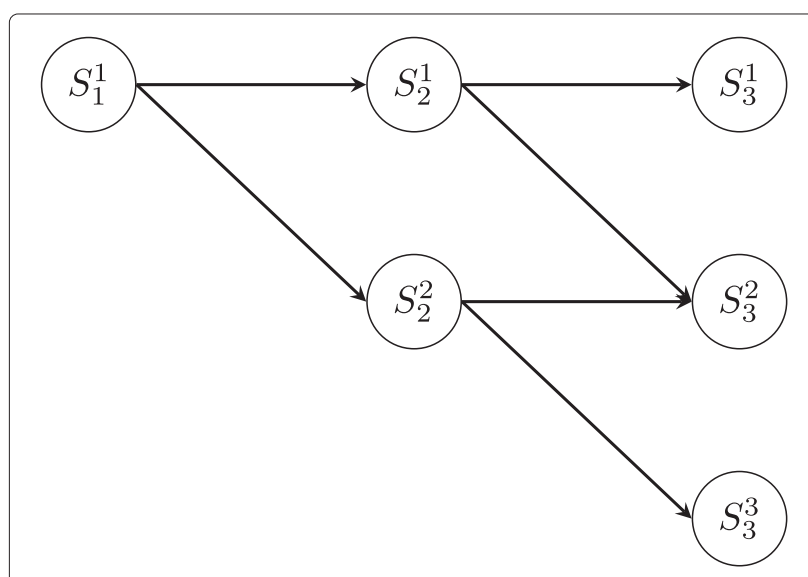

Fig. 3 Possible scenarios in the second and third time slots where $\mathrm{P}\left[T_{\mathrm{I}}(1)=1\right]$ is the probability of having $T_{\mathrm{I}}(1)=1$. In (10), we have accounted for the possibility of ending the contention round at the end of the first slot by multiplying $\left(1-\mathrm{P}\left[T_{\mathrm{I}}(1)=1\right]\right)$. Since $T_{\mathrm{I}}(1)=1$ happens when only one user transmits in the first slot, it is easy to show that

$$
\mathrm{P}\left[T_{\mathrm{I}}(1)=1\right]=N p_{\text {init }}\left(1-p_{\text {init }}\right)^{N-1} \text {. }
$$

Now, let us consider an arbitrary time slot $m>2$. In this time slot, there are $m$ possible states (values) for the access probability at the users resulting from $m-1$ access probability states at time slot $m-1$ (Fig. 4). We denote these states by $S_{m}^{j}$ for $1 \leq j \leq m$. That said, the user's access probability at state $S_{m}^{j}$, denoted by $p(m, j)$, for any $m \geq 2$, can be written as

$$
p(m, j)= \begin{cases}p(m-1,1)+\alpha & j=1, \\ p(m-1, j-1)-k \alpha=p(m-1, j)+\alpha & 1<j<m, \\ p(m-1, m-1)-k \alpha & j=m .\end{cases}
$$

Note that in (12), $p(1,1)=p_{\text {init }}$. 


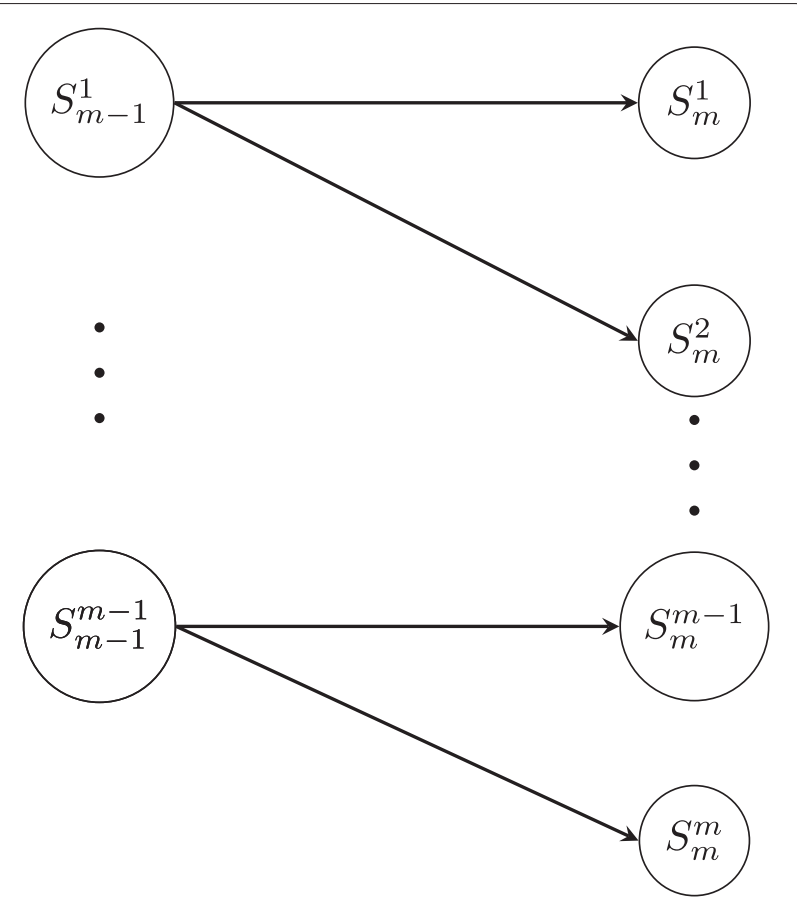

Fig. 4 Possible scenarios in time slot $m$

Similarly, the probability of being in state $S_{m}^{j}$, denoted by $q(m, j)$, for any $m \geq 2$, is

$q(m, j)= \begin{cases}q(m-1,1)(1-p(m-1,1)) & j=1 \\ q(m-1, j)(1-p(m-1, j))+q(m-1, j-1) p(m-1, j-1) & 1<j<m, \\ q(m-1, m-1) p(m-1, m-1) & j=m .\end{cases}$

Note that in (13), we have $q(1,1)=1$ meaning that a user always start from state $S_{1}^{1}$ at the begining of a contention round. Here, (13) comes from the fact that the user's access probability is in state $S_{m}^{j}$, when either its previous time slot state has been $S_{m-1}^{j-1}$, and it has had a transmission with probability $p(m-1, j-1)$, or it has been in the state $S_{m-1}^{j}$, and it has not transmitted with probability $1-p(m-1, j)$. Now, the average probability of access in time slot $m$ is

$$
\bar{p}(m)=\left(1-\sum_{i=1}^{m-1} \mathrm{P}\left[T_{\mathrm{I}}(i)=1\right]\right) \sum_{j=1}^{m} p(m, j) q(m, j) .
$$

Here, $\mathrm{P}\left[T_{\mathrm{I}}(i)=1\right]$ is the probability of having $T_{\mathrm{I}}(i)=1$. Multiplying $\left(1-\sum_{i=1}^{m-1} \mathrm{P}\left[T_{\mathrm{I}}(i)=1\right]\right)$ when calculating $\bar{p}(m)$ in (14) guarantees that we never have reached the instantaneous throughput of 1 up until time slot $m$.

Note that in deriving (12), (13), and (14), we have ignored the cases where the access probability at the user hits 0 or 1 . However, we claim that ignoring these cases does not noticeably affect $\bar{p}(m)$. As we see later, $\alpha$ has a very small value, hence, observing $p(m, j)=1$ is very unlikely. On the other hand, if for some state $S_{m}^{j}, p(m, j)$ hits 0 , since $k \geq 1$ and $\alpha \leq k \alpha$, states originated from $S_{m}^{j}$ in the following time slots will have a small access probability oscillating between 0 and a small positive value. As a result, these cases can be ignored when we want to evaluate $\bar{p}(m)$. For the values of $m$ where 0 or 1 are hit, to make sure that unaccepted probability terms (i.e., values less than 0 or greater than 1$)$ are avoided, we set $p(m, j)=q(m, j)=0$ if

$$
p_{\text {init }}+(m-j) \alpha-(j-1) k \alpha<0
$$

or

$$
p_{\text {init }}+(m-j) \alpha-(j-1) k \alpha>1 \text {. }
$$

In addition, to find $\bar{p}(m)$ from (14), one should find $\mathrm{P}\left[T_{\mathrm{I}}(i)=1\right]$. Deriving $\mathrm{P}\left[T_{\mathrm{I}}(i)=1\right]$ for a general $i$ is equivalent to finding the probability of having a full-rank $i \times i$ matrix whose elements are randomly selected (with different probabilities though) from $\{0,1\}$. Finding the rank of a matrix with random elements is quite challenging and requires a very involved derivation. That said, a closed-form solution for $\mathrm{P}\left[T_{\mathrm{I}}(i)=1\right]$ is significantly difficult to find. However, it can be verified through numerical analysis that $\mathrm{P}\left[T_{\mathrm{I}}(i)=1\right]$ for $i>3$ is often very small, e.g., in the order of $10^{-5}$ or even smaller for a typical value of $N$. Thus, we use the following approximation for $\bar{p}(m)$ in the rest of the paper:

$\bar{p}(m)= \begin{cases}p_{\text {init }} & m=1, \\ \left(1-\sum_{i=1}^{m-1} \mathrm{P}\left[T_{\mathrm{I}}(i)=1\right]\right) \sum_{j=1}^{m} q(m, j) p(m, j) & m=2,3, \\ \left(1-\sum_{i=1}^{3} \mathrm{P}\left[T_{\mathrm{I}}(i)=1\right]\right) \sum_{j=1}^{m} q(m, j) p(m, j) & m>3 .\end{cases}$

To evaluate $\bar{p}(m)$ from (17), it suffices to only analytically find $\mathrm{P}\left[T_{\mathrm{I}}(1)=1\right]$ and $\mathrm{P}\left[T_{\mathrm{I}}(2)=1\right]$. Here, $\mathrm{P}\left[T_{\mathrm{I}}(1)=1\right]$ is found according to (11). Further, it can be shown that

$$
\begin{aligned}
\mathrm{P}\left[T_{\mathrm{I}}(2)=1\right] & =2\left(\begin{array}{c}
N \\
2
\end{array}\right)\left(p_{\text {init }}\left(1-p_{\text {init }}\right)^{N-1}\right. \\
& \left.\times\left(p_{\text {init }}-k \alpha\right)\left(p_{\text {init }}+\alpha\right)\left(1-\left(p_{\text {init }}+\alpha\right)\right)^{N-2}\right) .
\end{aligned}
$$

Using the above analysis, we can derive a sufficient condition such that $\bar{p}(m)<\bar{p}(m-1)$. To this end, we only focus on $m>3$ which is of our interest ${ }^{6}$. That said, using (12), (13), and the average access probability given in (17) for $m>3$, one can show that

$$
\bar{p}(m)=1+(1-(k+1) \alpha) \bar{p}(m-1) .
$$


Now, to have $\bar{p}(m)<\bar{p}(m-1)$, the following condition should be satisfied:

$$
k>\frac{1}{\bar{p}(m-1)}-1
$$

While (20) gives a very simple condition for choosing $k$ (and hence simplifying the optimization in (9)), satisfying the condition on $k$ with a fixed step size becomes increasingly difficult as $k$ grows very fast with $m$. To overcome this challenges, we propose the second adaptive frameless ALOHA approach that benefits from variable step sizes to ensure (20) is satisfied.

\subsection{Adaptive probability of access with variable parameters}

To ensure that the condition in (20) is satisfied, the idea here is to let $k$ change adaptively (denoted hereafter by $k_{i}(m)$ for $\left.i \in \mathcal{N}\right)$, so that a decreasing $\bar{p}(m)$ is always guaranteed. That is, an arbitrary user $u_{i}$ updates its access probability as

$p_{i}(m)= \begin{cases}p_{i}(m-1)-\alpha_{i}(m-1) k_{i}(m-1) & \text { if } s_{i}(m-1)=1, \\ p_{i}(m-1)+\alpha_{i}(m-1) & \text { if } s_{i}(m-1)=0,\end{cases}$

for any $m \geq 2$. To ensure that $\bar{p}(m)$ is decreasing in $m$ and using $(20), k_{i}(m)$ is determined as

$$
k_{i}(m-1)=\left\lceil\frac{1-p_{i}(m-1)}{p_{i}(m-1)}\right\rceil .
$$

Further, to guarantee $0 \leq p_{i}(m) \leq 1$, we should have

$$
\alpha_{i}(m-1) \leq 1-p_{i}(m-1)
$$

and

$$
\alpha_{i}(m-1) \leq \frac{p_{i}(m-1)}{k_{i}(m-1)} .
$$

Satisfying the condition in (24) using fixed $\alpha_{i}(m)$ also becomes increasingly difficult as $m$ increases unless a very small fixed $\alpha_{i}(m)$ is chosen from the beginning. Choosing such a small value, however, may not give a good throughput or energy performance. Therefore, at the beginning of the contention round, all users set $\alpha_{i}(1)=\alpha$ where $\alpha$ is determined by the BS and sent to the users at the start of the contention round. Now, as time passes (i.e., $m$ increases), $\alpha_{i}(m)$ is set as follows

$$
\alpha_{i}(m-1)=\min \left\{\alpha, \beta \frac{p_{i}(m-1)}{k_{i}(m-1)}, 1-p_{i}(m-1)\right\}
$$

where $\beta \leq 1$ to satisfy (24). In this work, we assume $\beta=0.95$. Note that for any $m$, both $\alpha_{i}(m)$, and $k_{i}(m)$ are found locally at the users. Figure 5 summarizes our proposed schemes with variable step sizes.
To get the best energy saving out of the system, one can optimize the parameters of this approach. The optimization formulation is similar to (9); however, the optimization parameters are reduced to $\alpha, p_{\text {init }}$, and $F_{\mathrm{R}}{ }^{7}$. Thus, the intended optimization problem is formulated as

$$
\begin{array}{ll}
\min _{\alpha, p_{\text {init }}, F_{\mathrm{R}}} & d_{N} \\
\text { s.t. } & T \geq T_{\mathrm{o}} .
\end{array}
$$

The outputs of this optimization, $\alpha_{\mathrm{opt}}$ and $p_{\text {init }}$, are transmitted to the users by the BS at the start of a contention round. Also, $F_{\text {opt }}$ will be used as the stopping criterion for $F_{\mathrm{R}}$ at the BS. We call the throughput achieved via using these optimal parameters $T_{\mathrm{a}, \mathrm{v}}$.

\section{Simulation results}

In this section, we compare the original frameless ALOHA with our proposed adaptive access strategies in terms of both throughput and energy efficiency. The results of this section are obtained by averaging over 10,000 realizations of contention rounds for $N \in\{25,50,100\}$.

In our simulations, we compare several measures of these schemes. For throughput, we consider the average throughput of the system, denoted by $\bar{T}$. For original frameless ALOHA, first adaptive approach, and second adaptive approach, $\bar{T}$ is found by averaging $T_{\mathrm{o}}, T_{\mathrm{a}, \mathrm{f}}$, and $T_{\mathrm{a}, \mathrm{v}}$ over all simulation runs respectively. We call these average values $\bar{T}_{\mathrm{o}}, \bar{T}_{\mathrm{a}, \mathrm{f}}$, and $\bar{T}_{\mathrm{a}, \mathrm{v}}$. We also present the average number of transmissions by each users, denoted by $\bar{d}_{N}$, that is found by averaging $d_{N}$ over all runs.

In addition, we compare the average probability of transmission over all users and time slots, denoted by $p_{\mathrm{t}}$, for different schemes. Here, $p_{\mathrm{t}}$ is found as

$$
p_{\mathrm{t}}=\frac{\sum_{i=1}^{N} \sum_{m=1}^{M} p_{i}(m)}{M N} .
$$

We call the average of $p_{\mathrm{t}}$ over all simulation runs $\bar{p}_{\mathrm{t}}$. Another measure is $\bar{F}_{\mathrm{R}}$ that is the fraction of resolved users averaged over all the runs. Finally, we define $L=\frac{M}{N}$ representing the normalized length of a contention round. We also present the results for $\bar{L}$, the average of $L$ over all simulation runs.

In our simulations, SIC is executed at the BS to resolve the users' transmissions. Further, the optimization problem associated with each scheme is solved at the BS and the results are sent to the users at the start of the contention round. To solve the optimization problem for each value of $N$, we run a grid search over the optimization parameters for 10,000 times. The set of parameters that give the highest average for the objective function over these runs are then selected as the optimal parameters. For the grid search to optimize our proposed schemes, we use the parameters of the original frameless ALOHA 


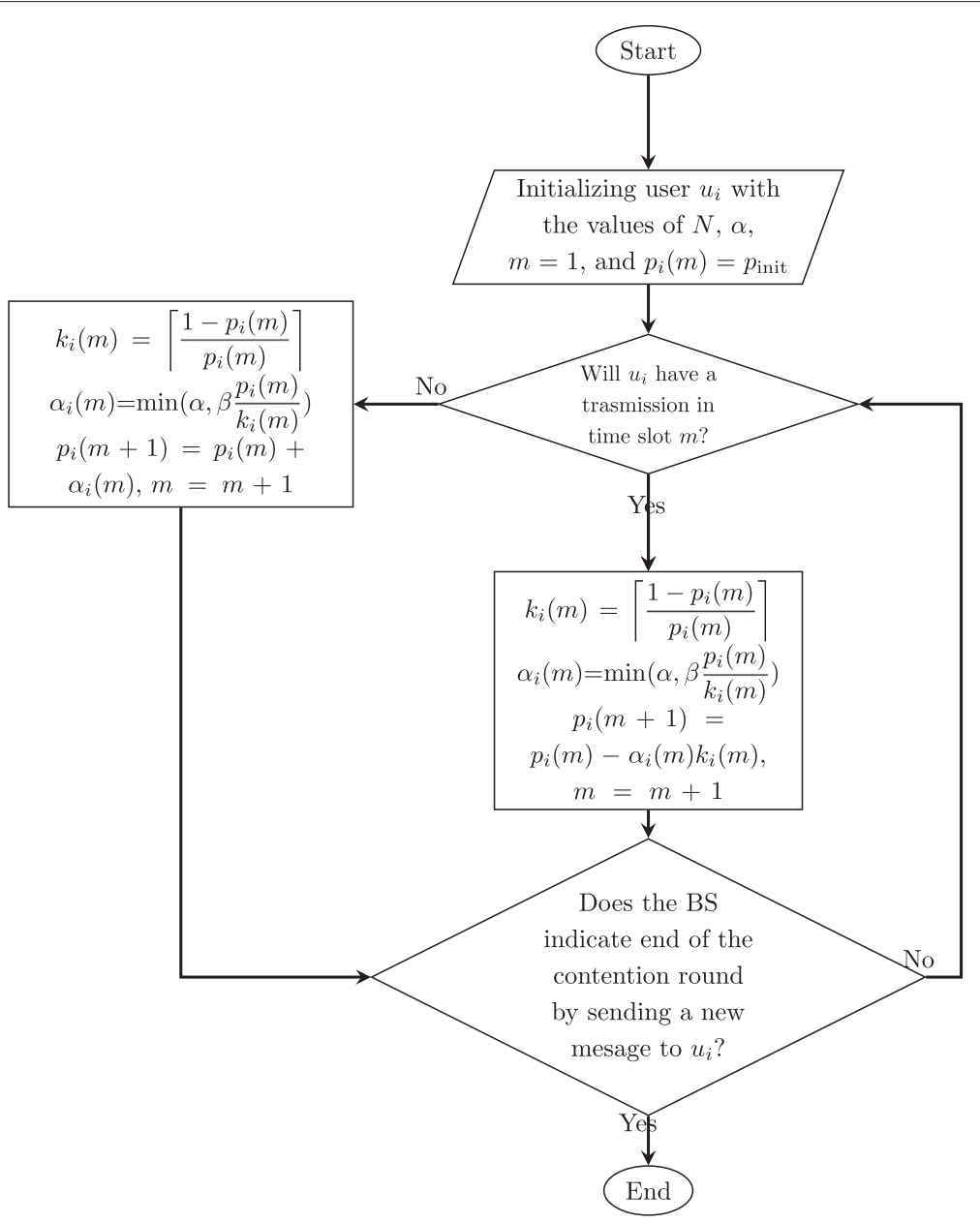

Fig. 5 Adaptive access strategy with variable step size

as a starting point. More specifically, to find the optimal values of $p_{\text {init }}$ and $F$ for our schemes, we search over $\left[p_{\text {init }_{\text {opt }}} / 2, p_{\text {init }_{\text {opt }}}\right]$ and $\left[F_{\text {opt }} / 2, F_{\text {opt }}\right]$ with step sizes 0.001 and 0.01 respectively where $p_{\text {init }}$ apt and $F_{\text {opt }}$ are the optimal values of $p_{\text {init }}$ and $F$ for the original frameless ALOHA derived in [17]. To find the optimal values of $\alpha$ and $k$ (for the first adaptive technique), we use an iterative grid search with adaptive mesh refinement to

Table 1 Performance of the original frameless ALOHA

\begin{tabular}{llll}
\hline$N$ & 25 & 50 & 100 \\
$p_{\text {init }}$ opt & 0.094 & 0.054 & 0.028 \\
$F_{\text {opt }}$ & 0.84 & 0.84 & 0.88 \\
\hline $\bar{T}_{\mathrm{O}}$ & 0.80 & 0.82 & 0.84 \\
$\bar{p}_{\mathrm{t}}$ & 0.094 & 0.054 & 0.028 \\
$\bar{d}_{N}$ & 2.32 & 2.68 & 2.68 \\
$\bar{F}_{\mathrm{R}}$ & 0.72 & 0.76 & 0.77 \\
$\bar{L}$ & 0.99 & 0.99 & 0.96 \\
\hline
\end{tabular}

improve the accuracy of the solution at each iteration. The search intervals for $\alpha$ and $k$ are $[0,0.5]$ and $[1,100]$ respectively. To search over $\alpha$ and $k$, we start by forming a two-dimensional regular coarse grid with only five points at each dimension, i.e., $\{0.1,0.2,0.3,0.4,0.5\}$ for $\alpha$

Table 2 Performance of adaptive frameless ALOHA with fixed step size

\begin{tabular}{llll}
\hline$N$ & 25 & 50 & 100 \\
$p_{\text {initopt }}$ & 0.082 & 0.049 & 0.024 \\
$F_{\text {opt }}$ & 0.83 & 0.86 & 0.88 \\
$\alpha_{\text {opt }}$ & 0.001 & 0.0001 & 0.00008 \\
$k_{\text {opt }}$ & 7 & 15 & 23 \\
$\bar{T}_{a, f}$ & 0.81 & 0.82 & 0.84 \\
$\bar{p}_{\mathrm{t}}$ & 0.085 & 0.049 & 0.025 \\
$\bar{d}_{N}$ & 2.03 & 2.36 & 2.38 \\
$\bar{F}_{\mathrm{R}}$ & 0.68 & 0.73 & 0.72 \\
$\bar{L}$ & 0.93 & 0.96 & 0.91 \\
\hline
\end{tabular}


Table 3 Performance of adaptive frameless ALOHA with variable step size

\begin{tabular}{llll}
\hline$N$ & 25 & 50 & 100 \\
$p_{\text {init }}$ opt & 0.081 & 0.044 & 0.025 \\
$F_{\text {opt }}$ & 0.81 & 0.92 & 0.89 \\
$\alpha_{\text {opt }}$ & 0.0027 & 0.0008 & 0.00011 \\
\hline $\bar{T}_{a, V}$ & 0.80 & 0.82 & 0.84 \\
$\bar{p}_{t}$ & 0.079 & 0.043 & 0.025 \\
$\bar{d}_{N}$ & 1.93 & 2.16 & 2.36 \\
$\bar{F}_{\mathrm{R}}$ & 0.7 & 0.75 & 0.75 \\
$\bar{L}$ & 0.98 & 0.99 & 0.95 \\
\hline
\end{tabular}

and $\{20,40,60,80,100\}$ for $k$. Then, we pick the point that maximizes the throughput over this coarse grid and form a (finer) grid in the vicinity of this point with again five points at each dimension. We continue searching and then refining the grid to the point that no significant change in the throughput is observed. We emphasize that the associated throughput with each of the grid points is found by averaging over 10000 runs. Note that if the number of users does not change between the contention rounds, which is often the case in moderately dynamic networks, same optimal value can be used for all contention rounds.

The simulation results for different schemes are presented in Tables 1, 2, and 3 as well as Fig. 6. By comparing the results in these tables, we can see that both adaptive schemes decrease the average number of transmissions in the network. This can be seen through the decrease

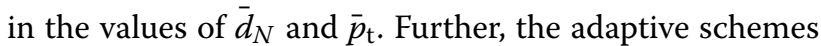
tend to use shorter contention rounds compared to the original frameless ALOHA and often terminate the contention round earlier. This is seen by comparing $\bar{F}_{\mathrm{R}}$ and $\bar{L}$ for the schemes.

The results for the energy consumption saving through our proposed schemes are presented in Table 4. The results in this table are derived by comparing $\bar{d}_{N}$ for the adaptive schemes with the one for the original frameless ALOHA. From this table, we can observe that both schemes provide a significant energy saving over original frameless ALOHA especially for small- and medium-sized networks. In addition, it can be seen that the adaptive scheme with variable step sizes results in a higher level of energy saving compared to the adaptive approach with fixed step sizes. For instance, when $N=50$, the second proposed adaptive approach achieves the outstanding amount of $19.5 \%$ in energy saving over the original frameless ALOHA. It is worth mentioning that the second approach achieves such a superior performance over the first approach with a lower optimization complexity. That is, allowing variable step sizes not only improves the energy efficiency but can also reduce the implementation complexity at the BS for an adaptive frameless ALOHA scheme.

Simulation results for $\bar{p}(m)$ is presented in Fig. 6 for $N=50$. For the original frameless ALOHA, users stick to a fixed transmission probability over all time slots. For the first adaptive approach, the average access probability sharply decreases at the beginning. However, for larger values of $m$, the condition in (20) is violated owing to

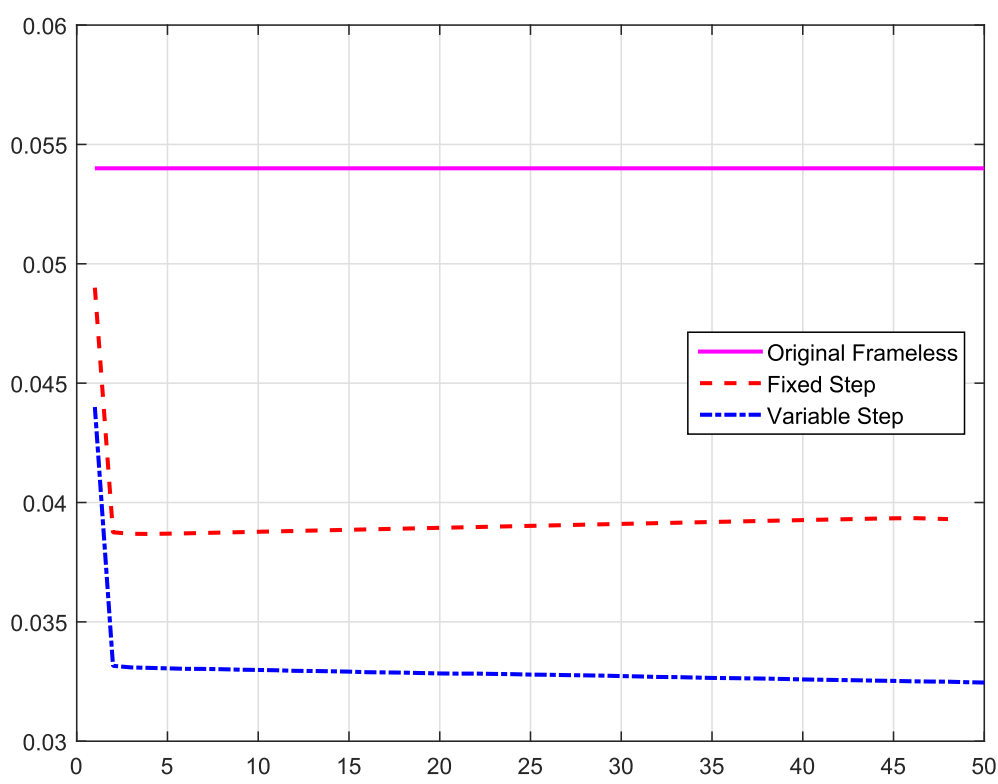

Fig. 6 Average access probability, $\bar{p}(m)$, of different schemes for $N=50$ 
Table 4 Improvement in the average energy consumption for the two proposed methods

\begin{tabular}{llll}
\hline$N$ & 25 & 50 & 100 \\
\hline Fixed step size & $12.5 \%$ & $11.9 \%$ & $11.2 \%$ \\
Variable step size & $16.8 \%$ & $19.5 \%$ & $11.9 \%$ \\
\hline
\end{tabular}

using a fixed $k$, and consequently, $\bar{p}(m)$ switches to a slow increasing trend. As seen in this figure, for the second adaptive approach, $\bar{p}(m)$ always has a decreasing trend over the contention round as a result of using variable step sizes.

\section{Conclusions}

In this work, we focused on improving the energy efficiency of frameless slotted ALOHA. To this end, we first proposed an adaptive scheme where the access probability at the users are adjusted with fixed step sizes to achieve better energy efficiency in the network. We then presented the analysis of the average access probability for this scheme. Using this analysis, a condition for ensuring a decreasing trend for the average access probability was derived. Difficulty of satisfying this condition through the adaptive scheme with fixed step sizes motivated us to propose our second adaptive approach where step sizes are variable. Through simulation results, we showed that the second approach results in a better energy saving over the first approach with even a lower computational complexity. The adjustments in the access probability at the users is done locally making our proposed schemes suitable for implementation in applications like RFID, M2M, and sensor networks. For future research direction, we will focus on addressing the effect of dynamic change in the number of users on our adaptive schemes and try to address this challenge accordingly.

\section{Endnotes}

${ }^{1}$ For dynamic networks where the number of users changes, an approach similar to the one proposed in [18] can be used to estimate $N$. An interested reader can also refer to [17] where the effect of imperfection in the estimation of $N$ has been studied on the performance of the frameless ALOHA.

${ }^{2}$ Frequency division or time division duplexing may be used to enable receiving the BS beacon message and transmitting packets by the users at each time slot. For more details, see [9].

${ }^{3}$ Our system model refers to higher layers where we are not concerned about the physical layer effects like fluctuation in the channel gain and noise.

${ }^{4}$ Similar phenomenon happens in decoding based on iterative belief propagation [19].
${ }^{5}$ This is assumed for the sake of simplicity and any positive real number $\beta>\alpha$ can be assumed as the decrease step.

${ }^{6}$ For a contention round, it happens very rarely to have $M \leq 3$. On the other hand, these occasional cases already have a good energy efficiency that cannot be further improved using our approach.

${ }^{7}$ Unlike (9), the BS does not need to optimize $k$.

\section{Competing interests}

The authors declare that they have no competing interests.

Received: 16 August 2015 Accepted: 9 August 2016

Published online: 17 August 2016

\section{References}

1. N Abramson, in Proceedings of the November 17-19, 1970, Fall Joint Computer Conference. The ALOHA system: another alternative for computer communications (ACM, New York, 1970), pp. 281-285

2. A Rajandekar, B Sikdar, A survey of mac layer issues and protocols for machine-to-machine communications. IEEE Internet Things J. 2(2), 175-186 (2015)

3. F Vazquez-Gallego, J Alonso-Zarate, L Alonso, in Proc. IEEE Intl. Conf. on Communications (ICC). Reservation dynamic frame slotted-ALOHA for wireless M2M networks with energy harvesting, (London, 2015), pp. 5985-5991

4. LG Roberts, ALOHA packet system with and without slots and capture. ACM SIGCOMM Comput. Commun. Rev. 5(2), 28-42 (1975)

5. H Okada, Y Igarashi, Y Nakanishi, Analysis and application of framed ALOHA channel in satellite packet switching networks-FADRA method. Electronics Commun. Jpn. 60, 72-80 (1977)

6. L Choudhury Gagan, S Rappaport Stephen, Diversity ALOHA-a random access scheme for satellite communications. IEEE Trans. Commun. 31(3), 450-457 (1983)

7. E Casini, R De Gaudenzi, OR Herrero, Contention resolution diversity slotted aloha (CRDSA): an enhanced random access scheme for satellite access packet networks. IEEE Trans. Wirel. Commun. 6(4), 1408-1419 (2007)

8. G Liva, Graph-based analysis and optimization of contention resolution diversity slotted ALOHA. IEEE Trans. Commun. 59(2), 477-487 (2011)

9. C Stefanovic, P Popovski, D Vukobratovic, Frameless ALOHA protocol for wireless networks. IEEE Commun. Lett. 16(12), 2087-2090 (2012)

10. JW Byers, M Luby, M Mitzenmacher, A Rege, A digital fountain approach to reliable distribution of bulk data. ACM SIGCOMM Comput. Commun. Rev. 28(4), 56-67 (1998)

11. ZG Prodanoff, Optimal frame size analysis for framed slotted ALOHA based RFID networks. Comput. Commun. 33(5), 648-653 (2010)

12. A Khodaian, BH Khalaj, Energy efficient transmission probability and power control in random access networks. (Citeseer), pp. 1-6. http://citeseerx.ist. psu.edu/viewdoc/summary?doi=10.1.1.140.3899

13. MA Bonuccelli, F Lonetti, F Martelli, in Proceedings of the 2006 International Symposium on on World of Wireless, Mobile and Multimedia Networks. Tree slotted ALOHA: a new protocol for tag identification in RFID networks (IEEE Computer Society, Niagara Falls/Buffalo, 2006), pp. 603-608

14. W Luo, A Ephremides, Power levels and packet lengths in random multiple access. IEEE Trans. Inf. Theory. 48(1), 46-58 (2002)

15. J Ahn, A Syed, B Krishnamachari, J Heidemann, Design and analysis of a propagation delay tolerant aloha protocol for underwater networks. Ad Hoc Netw. 9(5), 752-766 (2011)

16. M Luby, M Mitzenmacher, MA Shokrollahi, in Proceedings of ACM-SIAM Symposium on Discrete Algorithms. Analysis of random processes via and-or tree evaluation, vol. 98, (San Francisco, 1998), pp. 364-373

17. C Stefanovic, P Popovski, ALOHA random access that operates as a rateless code. IEEE Trans. Commun. 61(11), 4653-4662 (2013)

18. C Stefanovic, KF Trilingsgaard, NK Pratas, P Popovski, in Proc. IEEE Intl. Conf on Communications (ICC). Joint estimation and contention-resolution protocol for wireless random access, (Budapest, 2013), pp. 3382-3387

19. DJ MacKay, Fountain codes. IEE Proc. Commun. 152(6), 1062-1068 (2005) 